\title{
Redes sociales y relaciones digitales, una comunicación que supera el cara a cara
}

Social networks and digital relations, a communication that exceeds the face to face

\author{
Mireya Barón Pulido ${ }^{1}$ \\ Álvaro Duque Soto ${ }^{2}$ \\ Frederick Mendoza Lozano ${ }^{3}$ \\ Wilmar Quintero Peña ${ }^{4}$ \\ Politécnico Grancolombiano
}

Recibido: 13.12 .2020

Aceptado: 27.12.2020

\section{Resumen}

El presente artículo realiza una revisión bibliométrica de los estudios sobre medios sociales, y hace parte de la fase inicial de una investigación que busca caracterizar el consumo de medios sociales por parte de estudiantes de entre 14 y 17 años, de colegios privados de Bogotá. El proyecto es financiado por la Institución Universitaria Politécnico GrancolombianoColombia. El lector encontrará caracterizaciones que involucran la revolución digital, los estilos de vida y los medios y redes sociales (Wolfe, 2011). Nuestro propósito es orientarlo tanto frente a los conceptos hito que emergen tras la revisión bibliométrica (Sitios de Redes

\footnotetext{
${ }^{1}$ mbaronpu@poligran.edu.co https://orcid.org/0000-0002-8788-0519

https://scholar.google.com/citations?user=KRW10tEAAAAJ\&hl=es\&oi=ao

2 aduque@poligran.edu.co

https://scholar.google.com/citations?user=Nro0hXUAAAAJ\&hl=en

3 famendoza@poligran.edu.co https://orcid.org/0000-0001-5087-4476

https://scholar.google.com/citations?user=bMcsexgAAAAJ\&hl=en

4 jquinterop@poligran.edu.co https://orcid.org/0000-0002-6172-0453
} 
Social -SRS-, "Influencers") como frente a la ruta metodológica y la discusión de resultados. El artículo ofrece gráficos y dendogramas para tener una perspectiva sobre la tendencia que, en los últimos diez años, prevalece en la investigación sobre los medios sociales y su impacto en adolescentes, vistos desde lo pedagógico y comunicativo.

Palabras clave: Internet, Red social, Medios sociales, Tecnologías de Información y Comunicación.

\begin{abstract}
This article carries out a bibliometric review of social media studies, as part of the initial phase of research that seeks to characterize the consumption of social networks by 14-17-year-old students from private schools in Bogotá. The project is funded by the Institucion Universitaria Politecnico Grancolombiano-Colombia. The reader will find features involving the digital revolution and lifestyles, Social Media and Social Network (Wolfe, 2011), with the purpose of orienting him/her to the landmark concepts that resulted from the bibliometric review (Social Networking Sites -SRS-, Influencers), to the methodological path and to the discussion of results. The article also offers graphs and dendograms that allow a perspective on the trend that, in the last 10 years, from the pedagogical and communicative axis prevails in the research on social media and its impact on adolescents
\end{abstract}

Keywords: Internet, Social network, Social media, Information and Communication Technologie

\title{
1. Introducción y Estado de la cuestión
}

El uso de las Tecnologías de la Información y la Comunicación (TIC) en la educación ha planteado nuevas demandas a los educadores, los científicos de la educación y, en general, a todos los actores sociales. Las TIC constituyen la base de un sistema de enseñanza-aprendizaje 
que brinda aportes al establecimiento de nuevas metodologías pedagógicas y plantea transformaciones para el futuro de la educación.

Una de las más recientes manifestaciones de las TIC son los medios sociales, que, según lo propone Caivano (2010), se pueden entender como sistemas que permiten establecer relaciones de diversas maneras, vale decir construir y fortalecer las redes sociales, además de ser un espacio en el cual se da un intercambio de información. De esta manera, se reconoce una gran variedad de medios sociales a los que los adolescentes tienen acceso Instagram, Snapchat, Twitter, Google+, Vine, Tumblr. Por tal razón, el interés por incorporar los medios sociales en las instituciones educativas se ha incrementado dado que su uso en las aulas ha sido significativo e impacta el trabajo de los educadores y de las instituciones. En ese sentido, el estudio de Manca y Ranieri (2016) muestra que, en entornos de aprendizaje, tanto la información de uso formal como la de uso informal pueden emplearse en los procesos pedagógicos resultandos funcionales al momento de cumplir con el objetivo de enseñanza y aprendizaje Por ello, este análisis plantea la importancia de reconocer las múltiples y posibles relaciones que pueden presentarse en el contexto académico a partir del uso de los medios sociales en una población cuyas edades oscilan entre los 14 y 17 años.

De ahí la importancia de esta revisión de la literatura que permite identificar los conceptos ligados tanto a la educación y los medios sociales, como a los estudios relacionados con aspectos derivados del campo psicológico y clínico. Adicionalmente, la revisión realizada considera que es posible predecir la relación académica con los medios sociales en colegios conocer cuáles son las relaciones que permiten ofrecer herramientas para futuras investigaciones y aclarar dudas que han surgido con respecto al tema.

\section{La revolución digital y sus afectaciones en los estilos de vida}

La revolución digital puede entenderse como el crecimiento de la oferta de tecnología digital y la rápida sustitución de las tecnologías mecánicas y electrónicas por las digitales. Esta revolución digital influye en distintos aspectos de la vida de los individuos y las sociedades 
(Castells, 2009). Las aplicaciones y herramientas digitales son una visible manifestación de dicha revolución (Agar, 2019).

Como resultado de esa revolución, la forma de relacionarse de los seres humanos está presentando cambios importantes en diferentes rutinas y contextos cotidianos, esto es, en las formas de: establecer contacto, crear relaciones de amistad, planear viajes, realizar autovalidación y manejar procesos de aprendizaje, entre otros.

Autores como Agar (2019) comparan los efectos de las mediaciones tecnológicas en las relaciones humanas y la sustitución de interlocutores humanos por digitales con el comportamiento de los animales en un zoológico. Encuentran similitudes dado que, a pesar de recibir muchas calorías y la seguridad de una jaula que los aísla de cualquier depredador natural, existen claras manifestaciones de malestar psicológico en los animales. Esta observación de Agar (2019) es importante para el presente estudio pues muestra cómo las mediaciones digitales promueven una conexión menos directa entre humanos, dado que una sonrisa de un Emoji de Facebook, por ejemplo, no equivale a una sonrisa o cualquier expresión facial en vivo, para indicar un acuerdo sobre cualquier asunto. Así, un sinnúmero de interacciones, que antes cobraban sentido mediante la comunicación física del cara a cara, ha cambiado o se ha desplazado al contexto del plano digital.

El alcance de la relación entre las TIC y las redes sociales juveniles se refleja en diversos campos del desarrollo humano, tales como: el educativo, el sanitario, el psicológico, el político, el organizacional, el cultural y el ético, entre otros. Con la información que nos ofrece la bibliometría, se aprecia la diversidad de efectos que pueden llegar a tener las TIC sobre la forma como se gestan nuevas versiones de relaciones humanas. Diversos trabajos versan sobre los efectos de los medios sociales en los campos antes mencionados, con el objetivo de hacer notar la importancia de las redes como componente fundamental para el desarrollo social.

Existe una variedad de estudios que no han tenido en cuenta el efecto de las tecnologías, ocupándose solamente de las relaciones presenciales para analizar comportamientos sociales 
tales como la propensión a fumar, adoptar la bicicleta como medio de transporte, dedicar más horas a estudiar, etc. Lamentablemente, tales estudios no incluyen las redes sociales (mediaciones tecnológicas) que son uno de los instrumentos de la revolución digital. Es el caso de la investigación realizada en escuelas secundarias del norte de California (Ennett et al., 2008), en que se caracterizaron las relaciones entre pares adolescentes respecto de múltiples dimensiones, para determinar las causas de la propensión a fumar en adolescentes. Dicho estudio se centró en la valoración que los adolescentes hacen de fumar como un atributo personal para: involucrarse con compañeros, formar parte de la red de amigos que fuman, y condicionar la afiliación con amigos fumadores y/o relaciones familiares. Su resultado destaca que tener amigos fumadores es una variable altamente determinante en la propensión a fumar por parte de los adolescentes.

Por su parte, Goetzke y Tilmann (2011) analizan la relación entre las redes sociales presenciales y el capital social referido a la cultura de la bicicleta, el número de usuarios y la adopción de este medio de transporte. Mediante modelos probabilísticos encuentran que las redes sociales (presenciales) juegan un papel importante para viajes recreativos y, hasta cierto punto, para ir de compras. No hay evidencia que la variable capital social impacte sobre el aumento de viajes escolares y/o de trabajo (domicilios en bicicleta). La evidencia de los efectos de las redes sociales sobre los viajes recreativos no sorprende ya que el ocio implícito siempre tiene algún motivo social, tal como: la comodidad, la aprobación y la moda. Por otro lado, para ambos propósitos de viaje donde los efectos de las redes sociales son evidentes, la difusión de información según la cual el ciclismo funciona para una sociedad más sostenible puede ser un aspecto importante para las personas que eligen usar la bicicleta.

En relación con el efecto que suscitan las TIC sobre el capital social y el logro escolar, la literatura gira entorno a cómo se utilizan las TIC en los procesos de aprendizaje. Por su parte, el capital social funciona como el mecanismo por medio del cual se utilizan de manera positiva o negativa las TIC. En esta línea, Agasisti, Gil Izquierdo y Won (2017), con información de las pruebas PISA, muestran que los estudiantes que usan diariamente las TIC, sin tener control por parte de los padres para darles un uso correcto, sufren efectos negativos en sus actividades 
académicas, principalmente en países en desarrollo. De otra parte, Kang, Heo y Kim (2011) señalan que a la hora de estudiar la relación entre las TIC y el logro escolar es necesario incluir las competencias afectivas y socioculturales como objetivos escolares, no solamente los elementos académicos tradicionales (cognitivos). De hecho, encuentran una relación positiva entre las TIC y las competencias afectivas y socioculturales. Destacan el papel de las TIC en los procesos relacionales que existen entre los estudiantes en lo referido a su desarrollo escolar, y señalan que dichas tecnologías deben estar inmersas en las estrategias escolares.

Lahiry et al., (2019) estudian el efecto de los servicios de las redes sociales sobre las relaciones interpersonales y el logro escolar de un grupo de estudiantes de medicina en India, encontrando que más del $85 \%$ de los estudiantes usaban SRS (Servicios de redes sociales) para propósitos académicos y los concebían como elemento determinante en su rendimiento académico. Además, no encuentran efectos negativos en las relaciones con sus pares, lo que indica que los estudiantes eran conscientes de los valores éticos y sociales. Estos resultados en India son contrarios a la evidencia encontrada en población universitaria de EEUU y Sri Lanka, donde el uso de redes sociales afecta negativamente el rendimiento escolar y las relaciones con sus compañeros (Thuseethan \& Kuhanesan, 2014; Kirschner \& Karpinski, 2010; Habes, Alghizzawi, Khalaf, Salloum \& Ghani, 2018). De hecho, los autores mencionados encuentran que para los estudiantes universitarios resultan relevantes las diferencias entre usuarios y no usuarios de Facebook: los usuarios dedican menos tiempo semanal al estudio y obtienen menores calificaciones. Adicionalmente, Habes et al., (2018) muestran que Facebook y las redes sociales son fuentes de información y comunicación entre los estudiantes. El uso promedio resulta ser de 4 a 12 horas al día, confirmando que las redes sociales se han convertido en parte integral de su vida cotidiana.

Kuo et al., (2013) sugieren que la teoría de la auto representación puede explicar por qué los usuarios de Facebook se desempeñan como actores que están constantemente en actividades de manejo de la imagen personal. Lo que es consistente con la teoría de Goffman quien, con base en la teoría del capital social y cognitivo, muestra que, para mantener sus relaciones sociales, las mujeres usan más los medios sociales que la población masculina. 


\section{Hacia una caracterización del network: sitios de red social}

Con base en la evolución temática de los diversos estudios realizados entre los años 2011 y 2020, que recogen el legado de las investigaciones comprendidas entre 2000 y 2010 (ver figura 3 ), se destaca el concepto de redes sociales y contexto educativo. Tal es el caso de un gran número de estudios sobre el binomio "educación y tecnología”, y de los trabajos sobre los ecosistemas digitales que involucran el escenario del internet y otras plataformas, entre las que se incluyen los sitios de red social, SRS.

Para profundizar sobre la pesquisa realizada desde Bibliometrix, resulta pertinente destacar los rasgos que caracterizan una red social y su impacto en el contexto educativo, como lo sugiere la compilación de ensayos en "Los desafíos de las TIC para el cambió educativo", coordinada por Carneiro, Toscano y Diaz (2016). Entre los articulistas de dicha publicación, Mariano Segura (2015) destaca que un sitio de red social (SRS) es: “(...) aquella estructura social formada por nodos (habitualmente individuos u organizaciones) que están vinculados por uno o más tipos de interdependencia tales como valores, puntos de vista, ideas..." (Segura, 2015, p.106).

A su vez, Obdulio Martín (2016) enfatiza que es pertinente diferenciar las redes sociales de las comunidades sociales. Las comunidades suelen estar impulsadas por un tema u objetivo concreto; controladas por guías o moderadores (por tanto, son descendientes), y cuentan con una cierta arquitectura organizativa e incluso pueden estar encuadradas en un lugar o ámbito específico. Por su parte, las redes no están centradas en un lugar, sino en los individuos que intervienen en ellas; se auto organizan; poseen una dinámica aleatoria, y están controladas por el usuario.

En línea con esa distinción, vale la pena referir la confusión que se hace, con frecuencia, entre redes sociales (social networks, en inglés) y medios sociales (social media, en inglés). En tanto que las primeras se refieren a los grupos de individuos que comparten intereses y 
pasiones; las segundas hacen referencia al instrumento, al medio con el que pueden iniciarse o fortalecerse las primeras.

Las redes sociales corresponden al término que empezó a emplearse por antropólogos y sociólogos ingleses a mediados de los años 50 del siglo XX, para caracterizar y estudiar el conjunto de relaciones humanas que tienen un impacto duradero en la vida de un individuo y ofrecen unos patrones y colaboraciones contingentes para orquestar las soluciones en la vida cotidiana (Wolfe, 2011).

El concepto de redes sociales, que se consolida en el análisis social hacia los años 70 del siglo $\mathrm{XX}$, se ha empleado para estudiar los apoyos sociales a partir de aspectos estructurales (clases sociales y relaciones de parentesco y amistad) antes que funcionales (políticas y administrativas, por ejemplo), tal como lo expone Requena Santos (1989).

Los medios sociales, de acuerdo con la definición de Kaplan y Haenlein (2010), son "el conjunto de aplicaciones y programas informáticos construidos a partir de los principios ideológicos y tecnológicos de la Web 2.0, que permiten la creación e intercambio de contenido generado por el usuario" (Kaplan, 2010, p.3).

Al plantear la categoría de medios de auto comunicación de masas, Manuel Castells (2009) señala que los medios sociales corresponden a un elemento relativamente nuevo del espacio público de la comunicación, copado hasta hace poco de modo prevalente y casi exclusivo por los medios de comunicación convencionales (identificados en inglés como los mainstream media). A diferencia de estos, los medios sociales permiten procesar, almacenar y transmitir información sin restricciones de distancia, tiempo o volumen y, sobre todo, permiten la retroalimentación.

En el trabajo en el que se enmarca la búsqueda de información para este artículo, se buscaron textos en los que se asumieran como iguales los medios y las redes sociales. Sin embargo, nuestra investigación deja claro que los primeros son plataformas que, al tener una estructura 
que propicia la interacción, contribuyen a la creación y el fortalecimiento de los segundos, siendo completamente diferentes.

\section{Pares como Influencers y cartera de medios}

En la revisión bibliométrica realizada, encontramos que el estudio de Paluck, Shepherdb y Aronowc (2015) destaca dos conceptos pertinentes para el componente metodológico. Así, al realizar estudios sobre el poder de la influencia ejercida por los pares en los climas cambiantes de conflicto escolar, emergen los conceptos de:

Estudiantes como referente social: se basa en las teorías del comportamiento humano que explican cómo los individuos atienden al comportamiento de ciertas personas de su comunidad, para entender lo que es socialmente normativo y, en respuesta, ajustar su propio comportamiento.

Estudiantes semilla: alude a la creación de una variable dependiente, pues el grupo investigador impulsó un pequeño grupo de estudiantes para tomar una postura " $\mathrm{x}$ " acorde con el objeto de estudio de lo investigado.

Por otro lado, la investigación realizada por Chua \& Chang (2015) enfatiza que, tras una revisión del estado del arte, se evidenció que los pares también habían sido identificados como una importante fuente de influencia que afecta las interpretaciones de los mensajes de las y los jóvenes en los medios de comunicación e información social. Dicha conclusión resultó de la relectura de las entrevistas a profundidad realizadas en 2014 a la población estudiantil femenina. También se ratificó que todos los participantes clasificaron a Instagram como su mayor SNS (Social Networking Services) de uso frecuente, seguido de Facebook, Twitter y Tumblr (Krayer et al., 2008).

El uso intensivo de medios sociales por parte de jóvenes puede tener aplicaciones educativas. En ese sentido, el estudio de Manca E, Ranieri M. (2016), realizó una revisión crítica de 
artículos con el objetivo de identificar si las posibles pedagogías que ofrece la red social Facebook se han explorado de la manera adecuada. Las clasificaciones de los trabajos dependen de su enfoque: 1.uso formal en entornos formales de aprendizaje,2. uso informal en entornos informales de aprendizaje; y 3. uso en entornos de aprendizaje informal. El análisis de los trabajos muestra que la mayoría de las investigaciones corresponden a los enfoques 1 y 2 , con el 46,9 y el 46,3 por ciento, respectivamente.

Junto a esta tendencia de uso de redes sociales en la población escolar, vale la pena mencionar que los adolescentes de hoy no se enfocan típicamente en una única plataforma. La mayoría de los adolescentes mantiene una "cartera de medios sociales" de varios sitios seleccionados, como lo indican las tasas de uso en la encuesta del Instituto Pew: Instagram (52\%), Snapchat (41\%), Twitter (33\%), Google+(33\%), Vine (24\%), Tumblr (14\%), y otros medios sociales (11\%). Así lo explica el informe técnico de la encuesta sobre beneficios y riesgos que provoca el consumo de los nuevos medios digitales en niños/adolescentes, realizado por Reid, Radesky, Christakis y Moreno (2016).

\section{Metodología}

Comúnmente, el análisis bibliométrico se utiliza para caracterizar la producción bibliográfica de una temática específica. Estos trabajos cuantifican la producción publicada e indexada en bases de datos científicas. Dependiendo del interés del investigador, el trabajo se puede centrar en el impacto de las publicaciones, la exploración de los autores clave, las revistas más relevantes o el desarrollo de los subtemas y su relevancia. En el presente artículo, la bibliometría se centró en este último aspecto, dado que su desarrollo está enmarcado en la exploración de los referentes conceptuales más relevantes que pueden dar luces para responder a la hipótesis que se mencionó en la introducción.

La revisión bibliométrica se realizó en dos momentos, mediante la búsqueda de las palabras clave. La primera búsqueda estuvo motivada por el interés de los investigadores en conocer las tendencias de investigación en torno a los temas de medios sociales, jóvenes y colegios. 
Con base en los resultados, se seleccionó un segundo grupo de trabajos relevantes después de una búsqueda de trabajos ampliada con palabras clave de la categoría "social support" que correspondió al conjunto de palabras clave de mayor relevancia o interés investigativo. El análisis de estos trabajos nutrió el apartado de discusión.

\subsection{Selección de una base de datos, palabras clave y ventana de observación}

Los dos referentes de indexación internacionales más relevantes son ISI Web of Knowledge y SCOPUS. Los resultados que se presentan a continuación se lograron haciendo uso del paquete Bibliometrix, que se ejecuta empleando el software de acceso libre R; y con la base conceptual sugerida por Aria y Cocurullo (2017).

La selección de las palabras de búsqueda es una tarea esencial para el desarrollo de un análisis bibliométrico. Este trabajo implica un análisis especializado de tesauros y la validación de expertos. Para desarrollar la bibliometría del análisis de datos, se hicieron búsquedas en la que se combinan varios términos clave derivados de la pregunta de investigación, para el presente caso: ¿Cuáles son las prácticas de consumo de las TIC's que utilizan los estudiantes de grado 11 de los colegios privados y/o distritales de Bogotá, durante el 2020? Por lo tanto, la expresión de búsqueda en SCOPUS fue: "social network" and "teenager or school".

\subsection{Análisis de la estructura conceptual}

El propósito de este análisis es identificar los subtemas de la temática principal y agruparlos por similitudes, de acuerdo con el criterio de co-ocurrencia de palabras clave (Aria \& Cuccurullo, 2017). Para lograrlo, se parte de una matriz que cruza todas las palabras clave con los documentos, de manera que se vuelve relevante la aparición conjunta de palabras en los documentos.

Las palabras clave de las publicaciones se disponen en una matriz $X$ (palabras clave vs.

documentos), en donde $X_{i j}$ toma el valor de 1 (si la palabra clave $i$ se incluye en el documento j) o el valor de 0 (en caso contrario). A través de un Análisis de Correspondencia Múltiple $(\mathrm{ACM})$, se construye un plano reducido a dos dimensiones en el que las palabras se 
representan más cerca, en función de la similitud de sus distribuciones (Batagelj \& Cerinšek, 2013; Börner et al., 2003; Cobo et al., 2011). El ACM permite tanto un análisis exploratorio sin asumir restricciones sobre los datos, como una interpretación sencilla en la que se establecen clústeres de palabras clave, según su posición en el plano factorial de dos dimensiones (Cuccurullo et al., 2016).

\subsection{Métricas de centralidad y densidad}

El análisis de temas clave a través del criterio de coocurrencia se puede visualizar como una red. De esta manera, las palabras clave agrupadas en clústeres, por medio del algoritmo $k$ means, conforman grupos que adquieren densidad, cuando hay una alta co-occurencia de palabras clave dentro de un mismo clúster. De acuerdo con Cobo et al. (Cobo et al., 2011), esta métrica se interpreta como el nivel de desarrollo dentro de una temática. Por su parte, la centralidad mide el grado de interrelación de la palabra clave de una temática con palabras clave de otras temáticas.

El índice de equivalencia (Cobo et al., 2011) se define como $e_{i j}=c_{i j}^{2} / c_{i} c_{j}$ Donde $c_{i j}$ es el número de documentos en los cuales dos palabras clave $i$ y $j$ co-ocurren. Y $c_{i}$ y $c_{j}$ representan el número de documentos en los que cada uno aparece.

A partir de un índice de equivalencia, Callon et al. (Callon et al., 1991) interpretan las coocurrencia de palabras clave como una red. Por consiguiente, definen dos métricas clásicas: 1) la centralidad, que puede ser definida así: $c=10 * \sum e_{k h}$ Donde $k$ es una palabra clave que pertenece a un tema, y $h$ es una palabra clave que pertenece a otros temas, y 2) la densidad, que puede ser definida así: $d=100\left(\sum e_{i j} / w\right)$ Donde $i$ y $j$ son palabras clave que pertenecen a un mismo tema, y $w$ es el número total de palabras clave dentro del tema.

En un plano cartesiano, los cuadrantes se pueden representar así (en sentido horario, empezando por el cuadrante superior de la izquierda):

- El primero representa los temas de mayor desarrollo (alta densidad), que a su vez están aislados, es decir, son muy especializados (baja centralidad). 
- El segundo representa temas altamente desarrollados y transversales: estos son los "motores" de la investigación.

- El tercero representa temáticas de baja centralidad y densidad, es decir, o son muy nuevos o están decayendo en relevancia. Aun así, se debe tener en cuenta que este análisis se hizo con los trabajos más relevantes según el criterio de selección de SCOPUS.

- Finalmente, el cuarto cuadrante presenta los temas básicos (de baja densidad) y transversales.

Es relevante relacionar estas métricas de densidad y centralidad a través de la diacronía. La importancia de un enlace temático puede ser medida por los elementos que tienen en común los temas enlazados. De acuerdo con Cobo et al. (Cobo et al., 2011), el índice de inclusión se define así: sea $T^{t}$ el conjunto de temas detectados en el subperiodo $t$, donde $U \in T^{t}$ representan cada tema detectado en el superiodo $t$. Sea $V \in T^{t+1}$ cada tema detectado en el periodo $t+1$. Se dice que hay una evolución temática desde $U$ hacia $V$ si hay palabras clave que se presentan en ambas y están asociadas a las redes temáticas. Por lo tanto, $V$ puede ser considerado un tema evolucionado desde $U$. Las palabras clave $k \in U \cap V$ son consideradas un nexo temático o un nexo conceptual, y su nivel de importancia está dado por: $I=$ $\frac{\#(U \cap V)}{\operatorname{Min}(\# U, \# V)}$

\section{Análisis y Resultados}

Los resultados que se obtuvieron se pueden apreciar en la tabla 1, donde se especifica la frecuencia de palabras clave:

\section{Tabla 1. Frecuencia de palabras clave}

\begin{tabular}{|l|l|l|c|}
\hline \multicolumn{1}{|c}{ Palabras clave de autor } & Articulos & \multicolumn{1}{c|}{ Palabras clave de indexación } & Artículos \\
\hline SOCIAL NETWORKS & 468 & FEMALE & 733 \\
\hline SOCIAL NETWORK ANALYSIS & 184 & MALE & 705 \\
\hline
\end{tabular}




\begin{tabular}{|l|l|l|l|}
\hline SOCIAL NETWORK & 150 & HUMAN & 635 \\
\hline ADOLESCENTS & 91 & ADOLESCENT & 628 \\
\hline SOCIAL CAPITAL & 74 & SOCIAL NETWORK & 558 \\
\hline ADOLESCENCE & 73 & HUMANS & 423 \\
\hline SOCIAL MEDIA & 64 & SOCIAL SUPPORT & 397 \\
\hline FACEBOOK & 55 & ARTICLE & 396 \\
\hline TEENAGERS & 49 & CHILD & 361 \\
\hline EDUCATION & 46 & SOCIAL NETWORKING ONLINE & 299 \\
\hline INTERNET & 43 & PEER GROUP & 276 \\
\hline SCHOOL & 36 & STUDENTS & 235 \\
\hline CYBERBULLYING & 34 & ADOLESCENT BEHAVIOR & 196 \\
\hline SOCIAL NETWORK SITES & 34 & ADULT & 190 \\
\hline YOUTH & 34 & EDUCATION & 181 \\
\hline FRIENDSHIP & 32 & SCHOOLS & 172 \\
\hline ADOLESCENT & 31 & SCHOOL & 164 \\
\hline SCHOOLS & 31 & MAJOR CLINICAL STUDY & 158 \\
\hline SOCIAL SUPPORT & 31 & PSYCHOLOGY & 151 \\
\hline CHILDREN & 30 & STUDENT & 142 \\
\hline
\end{tabular}

Estructura conceptual: En la estructura conceptual se pueden apreciar de manera específica las relaciones de los términos encontrados, contando con tres diferentes clústeres:

- Clúster 1 (Verde): Recoge estudios específicos entre las relaciones de amistad a través de estudios longitudinales.

- Clúster 2 (Azul): En el primer grupo, refleja la cercanía entre los términos con lo que se abarca el relacionamiento en jóvenes: relaciones entre grupos de pares o semejantes, relaciones humanas e interpersonales. Un segundo grupo de palabras clave están relacionadas con la metodología y los actores que son objeto de estudio en el análisis de redes sociales. Principalmente, escuelas, niños, jóvenes, jóvenes adultos, mujeres. De otro lado se recogen las palabras que describen estudios por medio de cuestionarios, experimentos humanos, análisis de redes sociales y ubica este tipo de estudios en el campo de estudio de la psicología.

- Clúster 3 (Rojo): En él se reflejan las palabras clave de los estudios enfocados en las relaciones entre medios sociales (ej. Facebook), el uso de internet y la educación, como se observa en la figura 1: 
Figura 1. Mapa temático

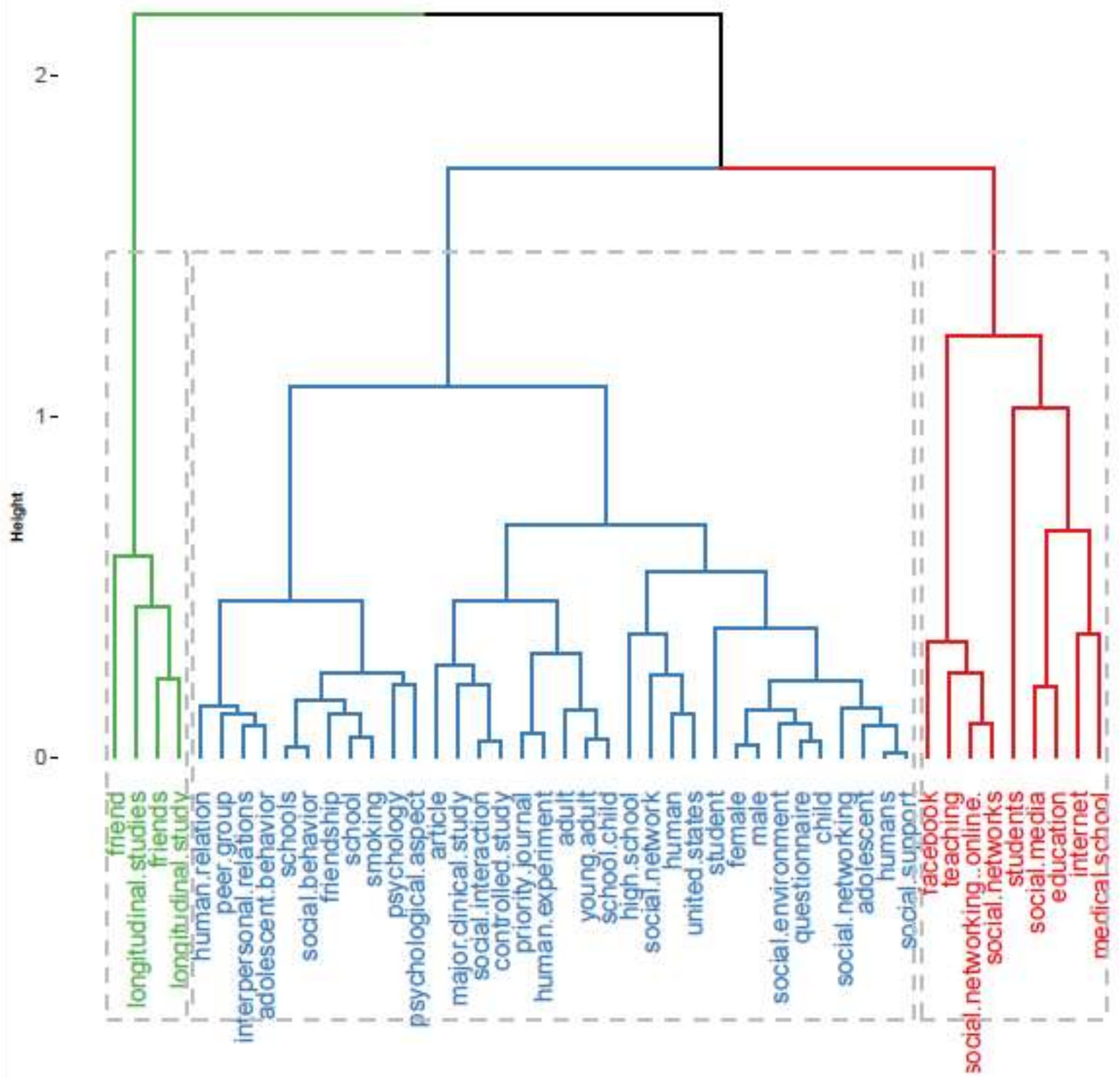


En el mapa temático se destacan los siguientes asuntos:

- Temas motores (mayor centralidad y densidad): Bajo la etiqueta "social support", se incluyen los temas de mayor cohesión e influencia en la red temática: que se remiten al estudio de las relaciones interpersonales, las condiciones sociales del entorno, aspectos psicológicos, la capacidad de afrontar situaciones difíciles en función del autoconcepto de los jóvenes, la autoestima y la motivación.

- Temas especializados (alta densidad y baja centralidad): Se refieren a los aspectos de salud física y mental en los jóvenes. Como se expuso en la sección de revisión de literatura, los trabajos de mayor relevancia tratan sobre las relaciones causales del uso de medios sociales con los desórdenes psicológicos y fisiológicos de los jóvenes, con especial énfasis en los efectos negativos derivados del cyber-bulling.

- Temas transversales: Bajo la etiqueta "human" se agrupan los temas de mayor centralidad, incorporándose de manera transversal en toda la temática de uso de medios sociales, e internet en jóvenes y colegios. Como descubrimiento relevante, están las palabras que reflejan la transversalidad los estudios de género y estudios controlados o experimentales.

- Temas de centro (nivel medio de centralidad y densidad): Con la etiqueta "humans" se agrupan las palabras clave de mayor pertinencia para el objetivo y la validación de Hipótesis de esta investigación. Allí se encuentra la palabra clave que se relaciona con los estudios en la escuela y dentro de eso las relaciones entre pares o jóvenes similares y los estudios con cuestionario para explorar el comportamiento social en redes sociales, como se sugiere en Tabla 2:

\section{Tabla 2. Comportamiento social en redes sociales}

\begin{tabular}{|c|c|c|c|c|}
\hline Centrality & Density & Name & Freq & Words \\
\hline 521,8523 & 32,09608 & Human & 5504 & $\begin{array}{l}\text { Human; social network; female; male; } \\
\text { article; adolescent; child; major clinical } \\
\text { study; controlled study; adult; student }\end{array}$ \\
\hline 417,7918 & 34,10343 & Humans & 2811 & $\begin{array}{l}\text { Humans; schools; school; peer group; } \\
\text { psychology; questionnaire; }\end{array}$ \\
\hline
\end{tabular}




\begin{tabular}{|r|c|l|l|l|}
\hline $\mathbf{6 8 8 , 9 8 9 3}$ & 101,5172 & Social support & 2294 & $\begin{array}{l}\text { adolescent behavior; social networking; } \\
\text { friend; social behavior }\end{array}$ \\
\hline $\mathbf{3 2 9 , 6 1 6}$ & 33,34397 & $\begin{array}{l}\text { Social support; interpersonal relations; } \\
\text { human relation } \\
\text { united states; social environment; } \\
\text { psychological aspect; interview; self- } \\
\text { concept; motivation; socioeconomic } \\
\text { factors }\end{array}$ \\
\hline & $\begin{array}{l}\text { Setworking } \\
\text { (online) }\end{array}$ & 2797 & $\begin{array}{l}\text { Social networking (online); students; } \\
\text { education; internet; teaching; social } \\
\text { networks; social media; Facebook; } \\
\text { medical school; } \\
\text { social sciences computing }\end{array}$ \\
\hline $\mathbf{3 7 2 , 5 1 6 6}$ & 43,67698 & $\begin{array}{l}\text { Health } \\
\text { behavior }\end{array}$ & 729 & $\begin{array}{l}\text { Health behavior; obesity; health survey; } \\
\text { health promotion; physical activity; } \\
\text { school health service; randomized } \\
\text { controlled trial; body mass; health } \\
\text { Education; exercise; Australia; public } \\
\text { health. }\end{array}$ \\
\hline
\end{tabular}

En la figura 2 se aprecia por predominancia de dichos clústeres, el eje central --color morado-. Tanto en la figura 2 como en la tabla 3 , se reconocen, tanto desde la densidad como desde la centralidad, 5 temáticas relevantes: Humanos (eje central), comportamiento humano, ayuda social (plano superior); y redes sociales y ser humano (Plano inferior):

Figura 2. Predominancia de clústeres

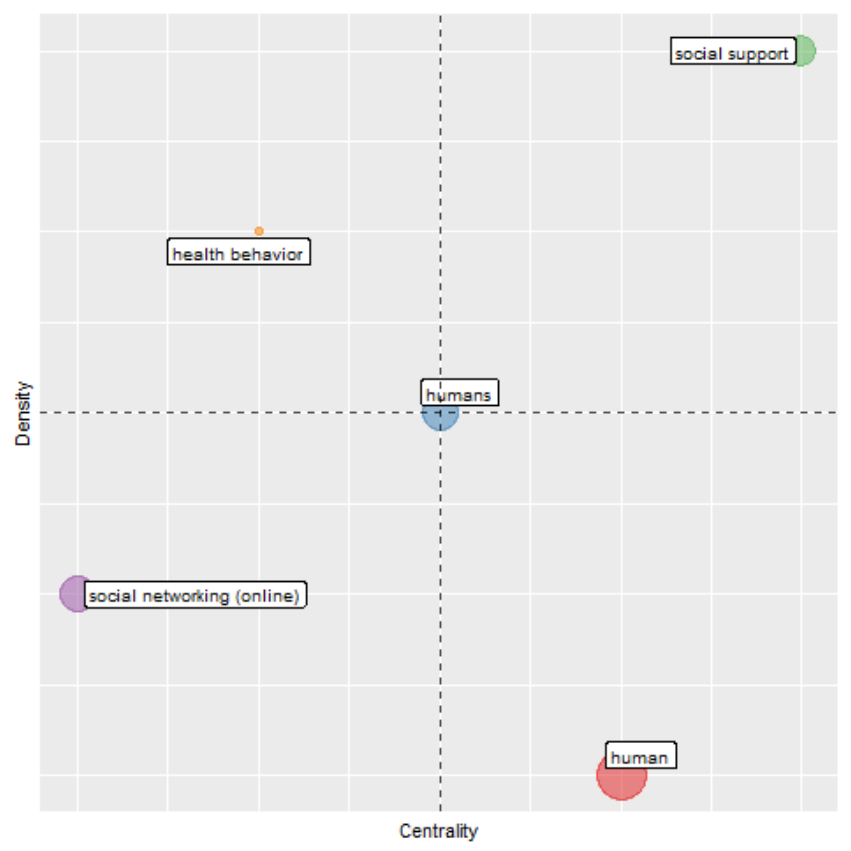


En la tabla 3, se precisa el paquete de palabras clave que acogen a cada una de estas 5 temáticas relevantes:

\begin{tabular}{|c|c|c|c|c|}
\hline Centrality & Density & Name & Freq & Words \\
\hline 521,8523 & 32,09608 & Human & 5504 & $\begin{array}{l}\text { Human; social network; female; male; } \\
\text { article; adolescent; child; major clinical } \\
\text { study; controlled study; adult; student }\end{array}$ \\
\hline 417,7918 & 34,10343 & Humans & 2811 & $\begin{array}{l}\text { Humans; schools; school; peer group; } \\
\text { psychology; questionnaire; } \\
\text { adolescent behavior; social networking; } \\
\text { friend; social behavior }\end{array}$ \\
\hline 688,9893 & 101,5172 & $\begin{array}{l}\text { Social } \\
\text { support }\end{array}$ & 2294 & $\begin{array}{l}\text { social support; interpersonal relations; } \\
\text { human relation } \\
\text { united states; social environment; } \\
\text { psychological aspect; interview; self- } \\
\text { concept; motivation; socioeconomic factors }\end{array}$ \\
\hline 329,616 & 33,34397 & $\begin{array}{l}\text { Social } \\
\text { networking } \\
\text { (online) }\end{array}$ & 2797 & $\begin{array}{l}\text { Social networking (online); students; } \\
\text { education; internet; teaching; social } \\
\text { networks; social media; Facebook; medical } \\
\text { school; social sciences computing. }\end{array}$ \\
\hline 372,5166 & 43,67698 & $\begin{array}{l}\text { Health } \\
\text { behavior }\end{array}$ & 729 & $\begin{array}{l}\text { Health behavior; obesity; health survey; } \\
\text { health promotion; physical activity; school } \\
\text { health service; randomized controlled trial; } \\
\text { body mass; health Education; exercise; } \\
\text { Australia; public health } 18\end{array}$ \\
\hline
\end{tabular}

La evolución temática se sugiere en la figura 3, donde se ilustra que en el primer periodo de observación (1970-2000), las palabras clave asociadas a educación migraron hacia casos que enfatizan en dos categorías en el segundo periodo (2001-2010): estudios sobre medios sociales y estudios humanos relacionados con aspectos clínicos o psicológicos 


\section{Figura 3. Evolución temática}

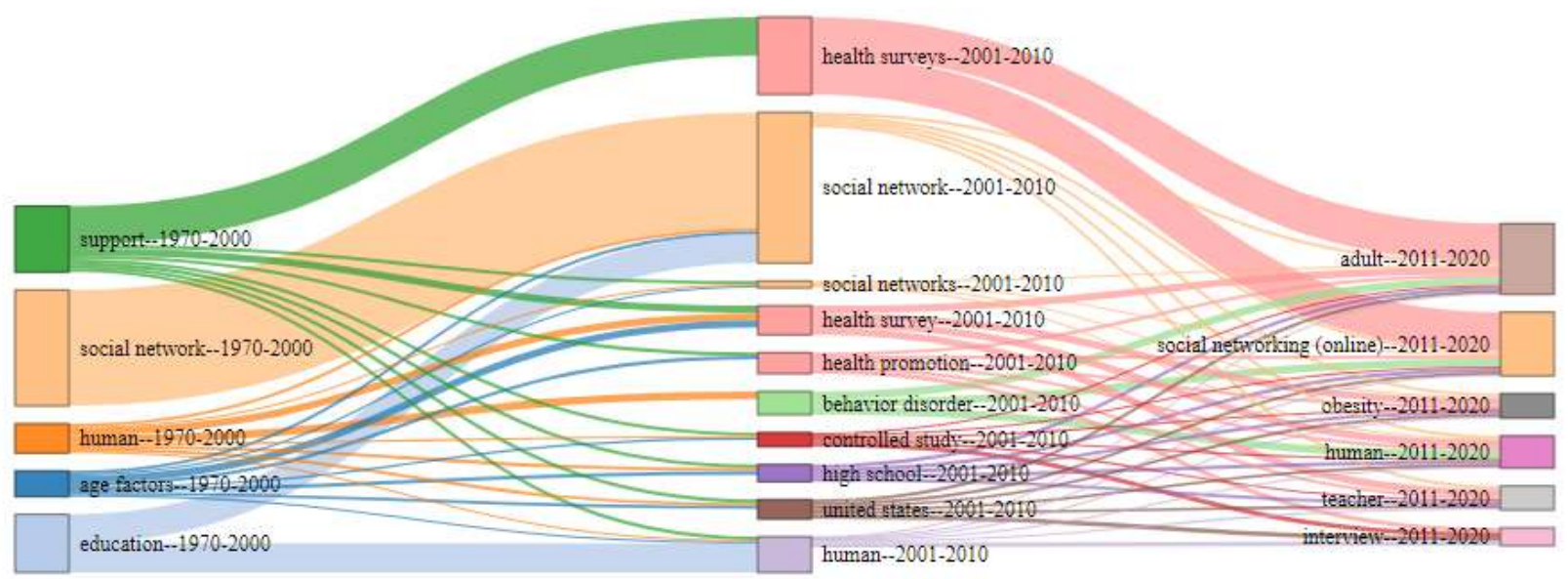

\section{Conclusiones}

Teniendo en cuenta la relevancia del término "social support", el equipo de investigadores realizó una segunda revisión literaria. Se perfiló la búsqueda a partir de las siguientes palabras clave: TITLE-ABS-KEY: "Social support" "interpersonal relations" "human relation" "social networks" "teenagers" OR school.

Los estudios sobre el relacionamiento suelen conducir a implicaciones psicológicas. Trabajos como el de Ranzini y Lutz (2016) indagan por la propensión a desarrollar rasgos narcisistas y/o a desvanecer las identidades individuales cuando se establecen relaciones a través de plataformas tecnológicas de citas explícitas, en las que la primera aproximación se consigue por medio de imágenes o vídeos de presentación.

Los estudios de Manago et al. (2012) brindan una valiosa pauta metodológica para el análisis del tipo de compensación que brinda Facebook en el desarrollo de la intimidad y el apoyo social, en una generación actual de adultos emergentes, es decir, la población universitaria de UCLA-EE. UU. El estudio diferenció 7 aspectos a medir en el diseño de instrumentos aplicados a través de la herramienta survey monkey: 
. Tamaño de la red

. Categorías de amigos (Medición de dichas categorías)

. Comunicación con los amigos de Facebook

. Actualizaciones de estado

. Satisfacción de la vida

. Autoestima

. Percepción de apoyo social en línea

Los datos de dicho estudio evidencian la estrecha relación, casi interdependencia, entre las bondades que brinda interactuar con una plataforma como Facebook y su impacto sobre las relaciones psicosociales. Impacto que se considera favorable dado que allí se satisfacen las relaciones psicosociales, dentro del actual ecosistema digital dinámico, cambiante y ágil en que consiste el dinámico mundo universitario.

Los hallazgos de dicho estudio también sugieren que los sitios de redes sociales ayudan a los jóvenes a satisfacer las necesidades psicosociales humanas, pues pretenden crear relaciones permanentes en un mundo geográficamente movedizo. Esto se refiere a los estudiantes universitarios cuyos contactos con amigos de la secundaria, principalmente, constituyen la mayor porción de los contactos que mantienen. De hecho, los jóvenes encuestados percibieron a Facebook como la herramienta más útil para obtener apoyo social (Manago, 2012).

La revisión literaria realizada sugiere a docentes, investigadores de la comunicación, pedagogos, psicólogos y diseñadores de currículo repensar el ecosistema digital en el que esta generación de adultos emergentes, es decir, la comunidad estudiantil universitaria, actúa, percibe, siente, comunica, interpela y cuestiona el día a día.

Esta primera revisión literaria sugiere tener presente tanto la relación como la diferenciación entre redes sociales (social networks, en inglés) y medios sociales (social media, en inglés): las primeras entendidas como los grupos de individuos; las segundas, como el factum mismo, esto es, la mediación tecnológica elegida por grupos específicos. 
Igualmente, sugiere diseñar estrategias didácticas multimediales, ilustrativas y preventivas que orienten a esta generación a interactuar con mesura en este contexto dinámico, cambiante. Un contexto que se transforma a una velocidad que a menudo supera nuestra capacidad de comprender los cambios, pero busca suplir todas y cada una de las necesidades psicosociales de los jóvenes universitarios. Por lo tanto, el diseño de estrategias es una misión en mora de ser realizada por parte de la comunidad de expertos que trabajan desde la interdisciplinariedad.

\section{Referencias}

Agar N (2019). How to Be Human in the Digital Economy. [Como ser humano en la economía digital] Cambridge, MA: MIT Press, 2018.

Agasisti, T., Gil-Izquierdo, M., y Han, S. W. (2017). ICT use at home for school related tasks: What is the effect on a student's achievement? Empirical evidence from OECD PISA data. [Uso de las TIC en el hogar y su relación con tareas escolares: ¿Cuál es el efecto en el desempeño del estudiante? Evidencia empírica de los datos del PISA de la OCDE. Munich Personal RePEc Archive (MPRA) Paper, no. 81343.

Aria, M., \& Cuccurullo, C. (2017). Bibliometrix: An R-tool for comprehensive science mapping analysis. [Bibliometría: Una herramienta $\mathrm{R}$ para la comprensión de análisis de mapas]. Journal of Informetrics, 11(4), 959-975.

Batagelj, V., \& Cerinšek, M. (2013). On bibliographic networks. [Sobre redes bibliográficas]. Scientometrics, 96(3), 845-864.

Börner, K., Chen, C., \& Boyack, K. W. (2003). Visualizing knowledge domains. [Visualización de dominios del saber] Annual Review of Information Science and Technology, $37(1), 179-255$. 
Caivano, Romina et al, (2010), Aplicaciones Web 2.0 Redes Sociales, Universidad Nacional de Villa María, Argentina, https://www.academia.edu/22586801/APLICACIONES_WEB_2.0_REDES_SOCIALES,

Callon, M., Courtial, J. P., \& Laville, F. (1991). Co-word analysis as a tool for describing the network of interactions between basic and technological research: The case of polymer chemistry. [El análisis de palabras como herramienta para describir la red de interacciones entre la investigación básica y la tecnológica: El caso de la química de polímeros]. Scientometrics, 22(1), 155-205.

Carneiro, Toscano y Diaz, Coordinadores (2016) Los desafíos de las TIC para un cambio educativo, OEI, Fundación Santillana.

Castells, M. (2009): Comunicación y poder. Madrid: Alianza.

Chua T, Chang L (2015), Follow me and like my beautiful selfies: Singapore teenage girls' engagement inself-presentation and peer comparison on social media. [Sígueme y Haz Like en mis hermosas selfies: El compromiso de las adolescentes de Singapur con su autopresentación y comparación entre pares en redes sociales. Journal Computers in Human Behavior, Elsevier, https://doi.org/10.1016/j.chb.2015.09.011.

Cobo, M. J., López-Herrera, A. G., Herrera-Viedma, E., \& Herrera, F. (2011). An approach for detecting, quantifying, and visualizing the evolution of a research field: A practical application to the fuzzy sets theory field. [Un enfoque para detectar, cuantificar y visualizar la evolución de un campo de investigación: Una aplicación práctica al campo de la teoría de conjuntos difusos. Journal of Informetrics, 5(1), 146-166.

Cuccurullo, C., Aria, M., \& Sarto, F. (2016). Foundations and trends in performance management. A twenty-five years bibliometric analysis in business and public administration domains. [Fundamentos y tendencias de la gestión de la actuación profesional. Un análisis 
bibliométrico de veinticinco años en los ámbitos de la empresa y la administración pública] Scientometrics, 108(2), 595-611.

Ennett, S.T., Faris, R., Hipp, J., Foshee, V.A., Bauman, K.E., Hussong, A., and Cai, L. (2008). Peer smoking, other peer attributes, and adolescent cigarette smoking: A social network analysis. [ Fumar entre pares, otros atributos de pares y fumadores adolescentes: Un análisis desde las redes sociales. Prevention Science, 9, 88-98.

Habes, M., Alghizzawi, M., Khalaf, R., Salloum, S. A., \& Ghani, M. A. (2018). The Relationship between Social Media and Academic Performance: Facebook Perspective. [ La relación entre las relaciones sociales, Medios de comunicación y rendimiento académico: Perspectiva desde Facebook]. International Journal of Information Technology and Language Studies.

Goetzke, Frank \& Rave, Tilmann. (2011). Bicycle Use in Germany: Explaining Differences between Municipalities with Social Network Effects. Urban studies (Edinburgh, Scotland). [ Uso de la bicicleta en Alemania: Explicación de las diferencias entre Municipios y efectos en redes sociales. Estudios urbanos Edimburgo, Escocia, https://doi.org/10.1177/0042098009360681

Kang, M., Heo, H. y Kim, M. (2011). The impact of ICT uses on new millennium learners' educational performance. [ El impacto del uso de la tecnología de la información y las comunicaciones en el rendimiento educativo de los estudiantes del nuevo milenio]. Interactive Technology and Smart Education, 8(1), 18-27.

Kaplan, Andreas y Haenlein, Michael. (2010). Users of the World, Unite! The Challenges and Opportunities of Social Media. [Usuarios del mundo, ¡uníos! Los desafíos y oportunidades de los medios sociales]. Business Horizons. 53. 59-68. 10.1016/j.bushor.2009.09.003. 
Kirschner, PA; Karpinski, AC. (2010). Facebook and academic performance. [Facebook y rendimiento económico]. Computer Human Behavior 26, 1237-1245

Kuo, F. Y., Tseng, C. Y., Tseng, F. C., \& Lin, C. S. (2013). A Study of Social Information Control Affordances and Gender Difference in Facebook self-presentation. [Un estudio de las asequibilidades de control de la información social y la diferencia de género en la autopresentación en Facebook]. Cyberpsychology, Behavior, and Social Networking, 16(9), 635-644

Lahiry, S., Choudhury, S., Chatterjee, S., \& Hazra, A. (2019). Impact of social media on academic performance and interpersonal relation: A cross-sectional study among students at a tertiary medical center in East India. [ Impacto de los medios sociales en el rendimiento académico y la relación interpersonal: Un estudio transversal entre los estudiantes de un centro médico terciario en la India Oriental]. Journal of education and health promotion, 8, 73. DOI: 10.4103/jehp.jehp_365_18

Levy Paluck, Elizabeth; Shepherd, Hana and Aronow, Peter M., (2016). Changing climates of conflict: A social network experiment in 56 schools, Cambiando los climas de conflicto: Un experimento de red social en 56 escuelas. Proc Natl Acad SciUSA, PNAS Journal, 113:566-571. https://doi.org/10.1073/pnas.1514483113

Manago A, Greenfield P, (2012), Me and My 400 Friends: The Anatomy of College Students' Facebook Networks, Their Communication Patterns, and Well-Being. [ Yo y mis 400 amigos: La anatomía de los estudiantes universitarios, Facebook, sus patrones de comunicación y el bienestar]. Developmental Psychology American Psychological Association Journal, Vol. 48, No. 2, 369-380 0012 1649/12/\$12.00, DOI:10.1037/a0026338

Manca, Stefania y Ranieri, María (2016). Facebook and the others. Potentials and obstacles of Social Media for teaching in higher education. [ Facebook y los otros potenciales y 
obstáculos de los medios sociales para la enseñanza en la educación superior]. Computers \& Education, v. 95, pp.216, 230. https://doi.org/10.1016/j.compedu.2016.01.012.

Martin Bernal, Obdulio (2016), Educar en comunidad: Promesas y realidades de la Web 2.0 para la innovación pedagógica, Los desafios de las TIC para el cambió educativo, OEI, Fundación Santillana, pp.78 - 9.

Reid Chassiakos, Radesky J, Christakis D, et al., (2016), AAP council on communications and media. Children and Adolescents and Digital Media. [ El consejo de la AAP sobre comunicaciones y los medios de comunicación. Los niños, adolescentes y los medios digitales]. Pediatrics. 2016;138(5): e20162593, DOI:10.1542/peds.2016-2593

Requena Santos, Félix (1989) El concepto de red social, REIS. Revista Española de Investigaciones Sociológicas, Núm. 48, Pág. 137-152

Segura, Mariano (2015), Plataformas educativas y redes docentes. Los desafíos d las TIC para un cambio educativo, OEI, Fundación Santillana, pp. 95-110

Thuseethan, S. y Kuhanesan, S. (2014). Influence of Facebook in Academic Performance of Sri Lankan University Students. [Influencia de Facebook en el rendimiento académico de Sri Lanka Estudiantes universitarios]. Global Journal of Computer Science and Technology, 14(4), 29-35. DOI: 10.2139/ ssrn.2478336

Van Dijk, Jan (2009): The Network Society. An Introduction to the Social Aspects of New Media, Londres: Sage.

Wolfe, Alvin. (2011). Anthropologist view of social network analysis and data mining. Visión antropológica del análisis de redes sociales y la minería de datos] Social Network Analysis and Mining. 1. 3-19. https://doi.org/10.1007/s13278-010-0014-4 
REVISTA INTERNACIONAL DE PEDAGOGÍA E INNOVACIÓN EDUCATIVA |

Volumen 1. Número 1. Enero - Junio 2021

ISSN: 2745-0341 (En línea) 